\title{
Management of traumatic brain injury patients
}

\author{
Hari Hara Dash ${ }^{1}$ and Siddharth Chavali ${ }^{2}$ \\ ${ }^{1}$ Department of Anesthesiology and Pain Medicine, Fortis Memorial Research Institute, Gurgaon, ${ }^{2}$ Department of \\ Neuroanesthesiology and Critical Care, All India Institute of Medical Sciences, New Delhi, India
}

Traumatic brain injury (TBI) has been called the 'silent epidemic' of modern times, and is the leading cause of mortality and morbidity in children and young adults in both developed and developing nations worldwide. In recent years, the treatment of TBI has undergone a paradigm shift. The management of severe TBI is ideally based on protocol-based guidelines provided by the Brain Trauma Foundation. The aims and objectives of its management are prophylaxis and prompt management of intracranial hypertension and secondary brain injury, maintenance of cerebral perfusion pressure, and ensuring adequate oxygen delivery to injured brain tissue. In this review, the authors discuss protocol-based approaches to the management of severe TBI as per recent guidelines.

Keywords: Guidelines; Management; Traumatic brain injury.

\section{Introduction}

Traumatic brain injury (TBI) is the leading cause of mortality and morbidity in patients in the age group of $18-45$ years. Most of the victims survive with significant disabilities, culminating in a major socioeconomic burden for both patients and their families. The financial burden of TBI is exorbitant. In the United States, in the year 2000 this was estimated to be $\$ 9.2$ billion in medical expenses and $\$ 51$ billion in lost productivity. In South Korea in 1998, the estimated number of head injury cases was 109,462 with an annual incidence of $236 / 100,000$ person, including 334/100,000 males and 136/100,000 females. The leading causes were road traffic accidents in $62.5 \%$ of cases and falls

Corresponding author: Hari Hara Dash, M.D., MNMS

Department of Anesthesiology and Pain Medicine, Fortis Memorial Research Institute, Sector - 44, Opposite HUDA City Centre, Gurgaon 122002, India

Tel: 91-9871153470, Fax: 91-1244921041

Email:dr.harihardash@gmail.com

ORCID: https://orcid.org/0000-0003-4020-5388

Received: January 3, 2018.

Revised: January 24, 2018.

Accepted: January 24, 2018.

Korean J Anesthesiol 2018 February 71(1): 12-21

https://doi.org/10.4097/kjae.2018.71.1.12 in $15.6 \%$ of cases [1].

The recent Brain Trauma Foundation (BTF) guidelines, published in 2016 [2], are protocol-based management strategies aimed at providing high quality care and improvements in outcomes for patients hospitalized with TBI. The cornerstone of the management of TBI is the intensive care treatment of these patients with careful attention paid to the airway, oxygenation and adequate hemodynamic support to avoid the secondary injuries that are associated with events such as hypoxia and hypotension. In this review, our aim and objective is to outline the management strategy of head injury patients based on the recent guidelines.

\section{Pathophysiology of TBI}

The initial stage after TBI results from direct tissue damage and impaired autoregulation of cerebral blood flow (CBF) along with disordered metabolism. This state, similar to ischemia, may lead to the accumulation of lactic acid, increased cell membrane permeability and subsequent edema. Since anaerobic metabolism cannot sustain the demands of the brain, Adenosine triphosphate (ATP) stores are depleted, which ultimately results in failure of the ATP-dependent membrane ionic pumps, which are essential for maintaining adequate homeostasis.

The second stage of this cascade is characterized by sustained

(c) This is an open-access article distributed under the terms of the Creative Commons Attribution Non-Commercial License (http://creativecommons.org/ licenses/by-nc/4.0/), which permits unrestricted non-commercial use, distribution, and reproduction in any medium, provided the original work is properly cited. 
membrane depolarization, along with excitotoxicity (i.e., the excessive release of excitatory neurotransmitters such as glutamate and aspartate) and the activation of voltage-dependent $\mathrm{Ca}^{++}$and $\mathrm{Na}^{+}$channels. The subsequent calcium and sodium influx results in the activation of lipid peroxidases, proteases and phospholipases, which trigger the apoptotic cascade and ultimately lead to membrane degradation and cell death.

Extracerebral organ dysfunction may be triggered and promoted by systemic inflammation following TBI. It has been suggested that the catecholamine surge following TBI is directly involved in the upregulation of cytokine levels, and may contribute to systemic organ dysfunction. Endocrine, respiratory and cardiac dysfunction (i.e., ST-T segment changes) are common, while renal and hepatic manifestations are unusual [3]. The most important of these organ dysfunctions in the acute setting is neurogenic pulmonary edema (NPE). NPE can develop immediately or during the 14 days following TBI. It leads to increased extravascular fluids in the lungs that cause hypoxia and decreased compliance [4]. The vascular tone of the pulmonary vessels is thought to increase as a consequence of the catecholamine storm that occurs after TBI and results in increased intravascular pressure and hydrostatic edema.

\section{Prehospital Management of TBI}

TBI is commonly classified into primary and secondary brain injuries. Primary brain injury involves damage to brain tissue resulting from the transfer of kinetic energy. Secondary brain injury is a term used to describe the aggravation of TBI over subsequent minutes to hours as a consequence of various factors, such as hypoxemia, hypotension, hypo- or hyper-carbia, hypo- or hyper-glycemia, hypo- or hyper-thermia, and seizures. Prevention of secondary brain injury is the primary concern of therapeutic interventions following TBI, which can best be managed by an anesthesiologist, preferably a neuroanesthesiologist.

Care of a TBI patient should begin at the site of the injury, with an aim to secure the patients' airway and maintain adequate ventilation and circulation [5] (Table 1). Patients with moderate or severe TBI should be transferred to a tertiary care center with neurosurgical facilities as soon as possible. Outcomes in TBI patients have been found to be influenced by transport methods, the duration of transit and whether the responding team is led by a physician or a paramedic. The primary management goals are the prevention of hypoxia and hypotension, because even a single episode of hypotension has been found to be associated with a doubling of mortality and an elevated risk of morbidity [6].

The Excellence in Prehospital Injury Care (EPIC) study, which began in 2014 [7], aims to enroll $\sim 25,000$ patients over the course of its study period. It aims to test the hypothesis that state-wide implementation of adult and pediatric emergency medical services TBI guidelines will significantly reduce mortality and morbidity in patients with moderate or severe TBI. A major focus is avoiding hyperventilation in the prehospital

Table 1. Protocols for the Prehospital Management of TBI

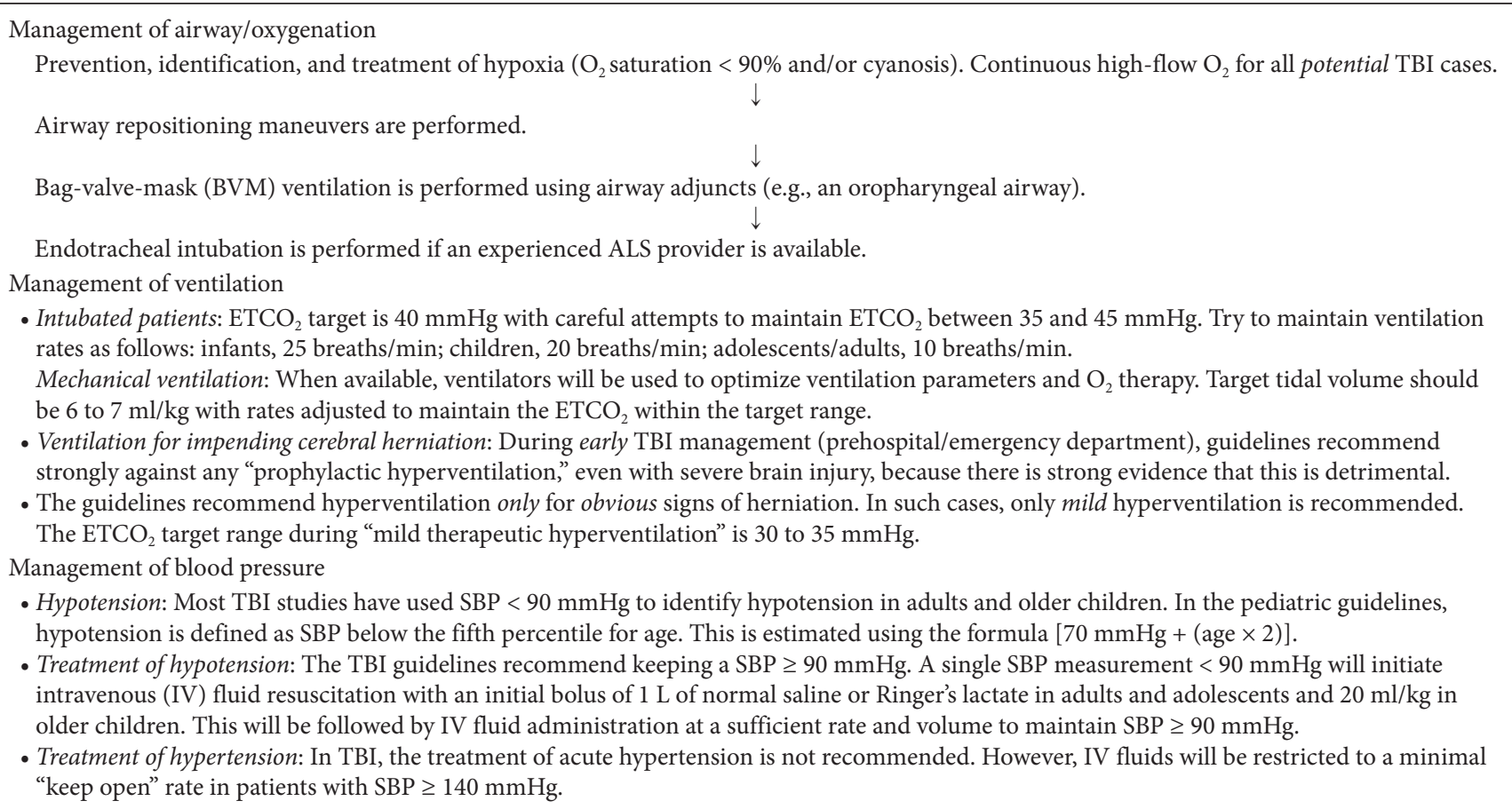


setting in TBI patients, as it has been found to lead to an approximately six-fold increase in poor outcomes [7].

\section{Neurological Assessment}

Since its original publication in 1974 by Teasdale and Jennett [8], the Glasgow coma scale (GCS) has been the most widely used method of recording the level of consciousness in patients at presentation and at subsequent assessments (Table 2).

The GCS has been criticized by some authors who state that the scale has poor inter-observer variability that leads to limited prognostic utility. Although there is no consensus, scales such as the simplified motor score and the Full Outline of Unresponsiveness score [9] have been developed as potential improvements to the GCS. Although they have higher inter-observer reliability, they have not yet found widespread acceptance.

A newer scale is the Rancho Los Amigos Scale [10], which is used to assess prognosis in patients following a closed head injury that is based on cognitive and behavioral patterns as a patient emerges from coma. It is often used in conjunction with the GCS because it can be used throughout the recovery period to track the progress of a patient. Patients are classed across eight grades, ranging from level I (no response) to level VIII (purposeful-appropriate).

\section{Airway Control and Ventilation}

Several studies have shown a correlation between hypoxemia and poor outcomes. Although airway control may be our primary concern in these patients, studies have reported poorer outcomes for TBI patients who were intubated at the site of trauma [11]. Intubation by inexperienced providers showed a four-fold increase in death and a significantly higher risk of worse functional outcomes when compared to patients whose airway was secured in the emergency department. In 2013, Sobuwa et al. [12] suggested that basic airway care performed well in a prehospital setting may be significantly better than prehospital intubation that is performed poorly.

Patients with TBI have up to a $5 \%$ to $6 \%$ incidence of an unstable cervical spine injury [13]. Risk factors include a motor vehicle accident and a GCS less than 8 . Therefore, all attempts at intubation should include in-line neck stabilization to reduce the chance of worsening a neurological injury until radiological clearance is obtained. Pre-existing hypoxia, intracranial hypertension, full stomach, and coexisting injuries, such as cervical spine trauma and maxillofacial injuries, may be present that predisposes a patient to difficult airway management. Thus, careful preparation and preoxygenation is mandatory.

Anesthetic drugs that allow for rapid control of the airway while avoiding an increase in intracranial pressure (ICP) and providing hemodynamic stability are preferred. Propofol and thiopental are the most commonly used drugs, but they may cause hypotension. Etomidate has advantages in terms of cardiovascular stability, but the possibility of adrenal suppression exists. Ketamine is popular in trauma patients and recent evidence suggests that its effect on ICP may be limited [14].

For rapid sequence intubation, succinylcholine or rocuronium may be used. Although succinylcholine may produce a small increase in ICP, this has to date not proven to be clinically significant. A retrospective study in 2016 compared rocuronium and succinylcholine in brain injured patients undergoing rapid sequence intubations in the emergency department. It reported an increased risk of mortality with succinylcholine; however, similar data from prospective studies are lacking. To obtain a response to laryngoscopy, an opiate such as fentanyl $(1 \mu \mathrm{g} / \mathrm{kg})$ may be used, but there is no evidence to support the use of lidocaine. Adequate sedation and muscle relaxation tends to reduce the cerebral metabolic oxygen requirement $\left(\mathrm{CMRO}_{2}\right)$, optimize ventilation, and prevent coughing or straining.

Ventilation of patients with severe TBI aims to maintain $\mathrm{PCO}_{2}$ within a normal range of 34-38 mmHg. Hypoventilation should be avoided, as increased $\mathrm{PCO}_{2}$ levels may lead to cerebral hyperemia with an increase in blood volume and ICP. Hyperventilation, on the other hand, results in an increased risk of vasoconstriction and increased tissue hypoxia, especially in the penumbra zone, so it is best avoided. The ongoing EPIC trial in Arizona focuses on avoidance of hyperventilation in TBI patients in a prehospital setting because it may lead to an approximately six-fold increase in poor outcomes. Hyperventilation up to a $\mathrm{PaCO}_{2}$ of $25 \mathrm{mmHg}$ for the purpose of reducing ICP is still accepted in the BTF Guidelines from 2016 for a brief period of time. Volume-guaranteed modes of ventilation may be a rational

Table 2. Glasgow Coma Scale

\begin{tabular}{|c|c|c|c|c|c|c|}
\hline & 1 & 2 & 3 & 4 & 5 & 6 \\
\hline Eye & Does not open & Opens to pain & Opens to voice & $\begin{array}{l}\text { Opens } \\
\text { spontaneously }\end{array}$ & & \\
\hline Verbal & No sounds & $\begin{array}{l}\text { Incomprehensible } \\
\text { sounds }\end{array}$ & Incoherent words & Confused & $\begin{array}{l}\text { Oriented, normal } \\
\text { speech }\end{array}$ & \\
\hline Motor & No movement & $\begin{array}{l}\text { Abnormal Extension } \\
\text { to pain }\end{array}$ & $\begin{array}{l}\text { Abnormal Flexion to } \\
\text { pain }\end{array}$ & Withdrawal to pain & Localizes pain & Obeys command \\
\hline
\end{tabular}


choice in these patients to minimize variations in $\mathrm{PaCO}_{2}$.

Fraction of inspired oxygen $\left(\mathrm{FiO}_{2}\right)$ settings on a ventilator should be adjusted to achieve a $\mathrm{PaO}_{2}$ of $\sim 90 \mathrm{mmHg}$, which can oxygenate the penumbra zone. High $\mathrm{PaO}_{2}$ should be avoided, considering the risk of hyperoxic cerebral vasoconstriction and hyperoxic lung injury. PEEP of 5-10 $\mathrm{cmH}_{2} \mathrm{O}$ may be administered to prevent atelectasis and has been proven to be safe in these patients [15]. Inhalation of beta-2 agonists may induce transient vasodilation with an increase in ICP and a reduction in blood pressure; if this occurs, the dose should be halved for subsequent inhalation therapy.

Evidence from recent trials indicates that although early tracheostomy may not be associated with a reduction in ventilator-associated pneumonia rates, ventilator/intensive care unit (ICU) days are reduced [16]. Consequently, the BTF has recommended that early tracheostomy should be performed to reduce ventilation days when the overall benefit outweighs the complications associated with the procedure (Level IIA).

\section{Blood Pressure and Cerebral Perfusion Pressure (CPP)}

Despite consensus on the principles of early management, there is no widespread agreement on resuscitation goals as various expert bodies have offered different management guidelines. Initially, the recommendation was to keep CPP above $70 \mathrm{mmHg}$ with vasopressors if needed. However, a subsequent study showed that outcomes were better with a relatively lower CPP, possibly because of a reduced incidence of Acute respiratory distress syndrome secondary to reduced vasopressor usage [17].

Vasopressors are commonly used to augment CPP in the setting of TBI, although data comparing these drugs is limited. Previous studies have found that norepinephrine had a more predictable and consistent effect on CPP, whereas dopamine use led to higher ICP levels [18]. Although there is minimal evidence to support the use of one vasopressor agent over another, a recent study suggested that phenylephrine may be associated with improved parameters [19].

The 4th edition of the BTF guidelines recommend:

1. Maintaining SBP at $\geq 100 \mathrm{mmHg}$ for patients 50 to 69 years old or at $\geq 110 \mathrm{mmHg}$ or above for patients 15 to 49 or over 70 years old to decrease mortality and improve outcomes (Level III);

2. The recommended target CPP value for survival and favorable outcomes is between 60 and $70 \mathrm{mmHg}$. Whether 60 or $70 \mathrm{mmHg}$ is the minimum optimal CPP threshold is unclear and may depend upon the patient's autoregulatory status (Level IIB);

3. Avoiding aggressive attempts to maintain CPP above 70 $\mathrm{mmHg}$ with fluids and pressors may be considered because

\section{Fluid Management}

In a hypotensive TBI patient, hypovolemia resulting from extracranial hemorrhage should be first ruled out. Even in the absence of extracranial hemorrhage, hypovolemia can still develop by trans-capillary leakage. Although it may be difficult to estimate the extent of hypovolemia, it can be roughly estimated using standard methods, such as analyses of the arterial pulse pressure variation, blood pressure response following a fluid challenge, or a passive leg raise.

Crystalloids are usually poor volume expanders, as 70-80\% reaches the interstitial space within 20 minutes of infusion, contributing to general systemic tissue edema. With a disrupted blood brain barrier (BBB), significant passive distribution into the brain interstitium may occur, leading to increased brain edema and increased ICP, especially if hypotonic solutions are used. Saline is the most common crystalloid used in TBI patients, but Ringer's lactate is an alternative. Infusion of large volumes of normal saline results in adverse hyperchloremic metabolic acidosis that is detrimental in TBI. Balanced crystalloid solutions may be a good alternative.

Colloids appear to provide no further benefit, and the SAFE trial found increased mortality in patients receiving albumin compared to saline [20]. TBI is associated with acute kidney injury in many patients (9-23\%) and often presents with consecutive higher mortality [21]. Colloids are also found to be associated with increased chances of acute kidney injury and increased use of renal replacement therapy in critical ill patients. Recent Cochrane reviews have demonstrated that the use of colloids is not superior to crystalloids for overall mortality, especially in patients with trauma, burns, or post-surgery [22]. However, administration in the setting of intact renal function may be considered.

Evidence seems to suggest that it is the volume of fluid infused, rather than the choice of fluid itself, that plays an important role in outcomes following TBI.

\section{Sedation and Analgesia}

Reducing stress and the adrenocortical response is an important component of TBI management [23]. Even unconscious TBI patients may have increased blood pressure and ICP resulting from this stress response. Sedative agents can reduce metabolic stress on acutely injured brain tissue by decreasing cerebral metabolism and consumption of oxygen in a dose-dependent manner [24] that, in turn, decreases CBF and leads to a reduction in ICP. Care should be taken to maintain an adequate mean arterial pressure throughout the duration of sedation.

Achieving an adequate level of sedation is paramount because it minimizes the length of hospital stay, ventilator days, 
incidence of delirium and helps in early mobilization [25]. The latest BTF recommendations regarding the use of sedatives and analgesics are as follows:

1. Administration of barbiturates to induce burst suppression as prophylaxis against intracranial hypertension is not recommended (Level IIB);

2. High dose barbiturates are recommended to control ICP refractory to maximum standard surgical and medical treatments while ensuring hemodynamic stability;

3. Although propofol may be used for ICP control, it is not recommended for improvements in mortality or sixmonth outcomes.

\section{ICP Monitoring and Management}

Patients with elevated ICP have been shown to have worse outcomes and are at a higher risk of mortality [26]. The indications of ICP monitoring in TBI from the latest edition of the BTF guidelines are as follows:

1. Management of severe TBI patients based on ICP monitoring may reduce in hospital and two-week post-injury mortality;

2. The guidelines no longer include a recommendation regarding patients that should be chosen for monitoring because of insufficient high quality evidence;

3. Clinical judgement should be used to initiate intracranial monitoring in patients who are at a high risk of clinical deterioration.

Updated BTF guidelines state that ICP monitoring is a level IIB recommendation, and recommend treatment of ICP > 22 $\mathrm{mmHg}$ to reduce mortality. The management of increased ICP includes standardized strategies that use a "staircase approach" with an escalating treatment intensity (Fig. 1). The American College of Surgeons TBI Guidelines recommend a three-tier approach for the management of increased ICP [27].

Invasive monitoring using the external ventricular drain (EVD) technique, in which a catheter is placed into one of the ventricles through a burr hole, is considered to be the gold standard of ICP monitoring. In addition to measuring ICP, this technique can also be used to drain cerebrospinal fluid and administer medicine intrathecally, such as for antibiotic administration in cases of ventriculitis. Additionally, EVD placement may be indicated to drain post-traumatic hemorrhage.

\section{Osmotherapy}

Osmotherapy with mannitol has been used since the 1960s as the main treatment for raised ICP and remains a component of TBI management guidelines. Hypertonic saline has become an alternative during the last 20 years, but controversy remains regarding which solution is the best agent and regarding the best method of administration. Mannitol increases CBF by plasma expansion, decreasing the blood viscosity via deformed erythrocytes, and promoting osmotic diuresis. Hypertonic saline promotes the flux of water across the BBB and improved blood flow by expanding the plasma volume. Cottenceau and colleagues compared equiosmolar doses of mannitol and hypertonic saline in the treatment of increased ICP [28]. The extent of ICP reduction from baseline was significantly higher in subjects treated with hypertonic saline compared with those treated with mannitol.

Because of limited evidence, the recommendations of the $3 \mathrm{rd}$ edition of the BTF guidelines were as follows: "Mannitol (0.25-1

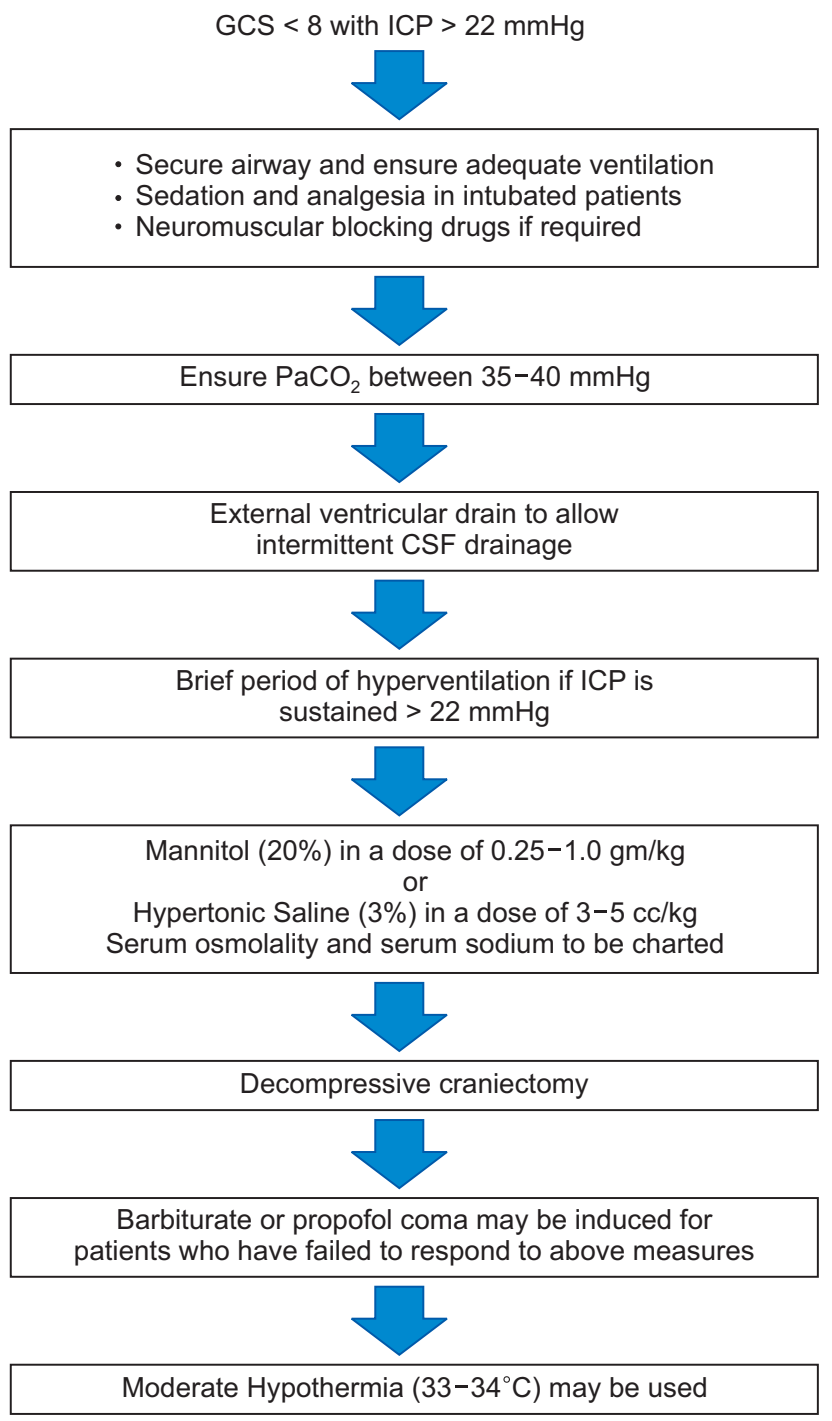

Fig. 1. Stepladder approach to the management of intracranial hypertension (ICP > $22 \mathrm{mmHg}$ ) after severe TBI (GCS < 8). CSF: cerebrospinal fluid, TBI: traumatic brain injury, GCS: Glasgow coma scale. 
$\mathrm{gm} / \mathrm{kg}$ ) is effective for the control of elevated ICP while avoiding hypotension (Level 1I)."

\section{Multimodal Neuromonitoring}

Identification of the range of autoregulation following TBI to provide individualized CPP therapy may be a means to improve outcome and is made possible by newer monitoring devices [29].

Jugular venous oxygen saturation $\left(\mathrm{SjvO}_{2}\right)$ can be used to estimate the balance between global cerebral oxygen delivery and uptake. A catheter is inserted in to the dominant internal jugular vein and advanced to the jugular bulb, aiming to minimize contamination from extracranial venous return. Reduction in jugular $\mathrm{SvO}_{2}$ levels below 55\% indicates that cerebral oxygen demand may be inadequate to meet demand, usually due to decreased CPP or hyperventilation associated vasoconstriction. Conversely, increased $\mathrm{SjvO}_{2}$ indicates excessive perfusion caused by raised $\mathrm{CBF}$ or decreased oxygen utilization secondary to cell death. Both reduction in $\mathrm{SjvO}_{2}<50 \%$ and $\mathrm{SjvO}_{2}>75 \%$ after TBI are associated with poor outcomes. Monitoring of $\mathrm{SjvO}_{2}$ following TBI may lead to improved outcomes [30].

The Licox monitor uses a closed polarographic electrode to measure focal brain tissue oxygen tension $\left(\mathrm{PbrO}_{2}\right)$. Oxygen diffuses across a semipermeable membrane and produces a current directly proportional to the oxygen concentration. The measured $\mathrm{PbrO}_{2}$ represents the balance between oxygen delivery and cellular oxygen consumption. $\mathrm{PbrO}_{2}$ provides a highly focal analysis of brain milieu and may be used to monitor the potentially salvageable penumbra following TBI. Normal values are between 35-50 mmHg. Following TBI, reduced levels of $\mathrm{PbrO}_{2}$ $(<5-10 \mathrm{mmHg})$ have been seen to be associated with poorer outcomes [31].

Cerebral microdialysis is being increasingly used as a bedside tool to provide analysis of brain homeostasis in the intensive care setting [32]. Severe ischemia is usually associated with significant increases in the lactate/pyruvate ratio (>20-25) and is associated with poor outcomes following TBI. Cellular lysis following TBI leads to degradation of membrane phospholipids and release of glycerol into extracellular fluid, making it a useful marker in this setting. Cerebral microdialysate concentrations of glycerol are typically elevated in the first 24 hours after TBI, followed by an exponential decline over the next 3 days. Subsequent increases in glycerol are associated with poor prognosis and seizure activity on electroencephalography (EEG) [33].

Posttraumatic seizures are a major cause of secondary brain injury following TBI, and are associated with higher injury severity and worse outcomes [34]. Recent data suggests that seizures occur in up to $20 \%$ of patients with TBI. These seizures are usually nonconvulsive in nature and cannot be detected clinically [35], making continuous EEG (cEEG) recordings a vital tool in diagnosis and management.

\section{Anticonvulsant Therapy}

Subsequent to TBI, convulsive activity results in increased ICP and altered oxygen supply to the injured brain. To prevent secondary brain injury, many studies have attempted to study the benefit of seizure prophylaxis. Temkin et al. [36] showed that treatment with phenytoin was effective in decreasing the rate of posttraumatic seizures in the first 7 days of injury, but had no significant role in prevention of posttraumatic seizures after the first week of injury.

Clinical comparisons of levetiracetam and phenytoin in prevention of early posttraumatic seizure prophylaxis have found no significant difference in rates of early posttraumatic seizures among patients treated with phenytoin compared with patients treated with levetiracetam [37]. The current BTF Guidelines recommend treatment with anticonvulsants within 7 days of injury. No randomized controlled studies have been performed till date to prove that one antiepileptic drug is better than another in this setting.

\section{Temperature Management}

In clinical practice, even mild hyperthermia has been associated with poorer outcomes and longer ICU stays, as it is may lead to increased edema and inflammation [38]. Conversely, cooling may be neuroprotective and has been seen to improve outcomes following global brain hypoxia. However, the traumatized brain suffers from compromised circulation and hypoxia in the penumbra zone, making it hypersensitive to the adrenergic stress induced by the brain injury itself, which may get aggravated active hypothermia.

The Eurotherm3235 Trial was designed to define the association of hypothermia and functional outcome in patients with TBI [39]. The trial was terminated prematurely, because signs of harm within the treatment arm were evident. Although there were statistically significantly fewer failures of therapy to control acutely increased ICP in the hypothermia group, the treatment group had lower extended Glasgow Outcome Scale scores at 6 months compared with the control group. O'Phelan et al. [40] reported a significant increase in pulmonary complications in patients treated with targeted temperature management compared with standard of care. This difference was explained by the inhibition of fever to fight infection.

The recommendation from the BTF is that early $(<2.5 \mathrm{~h})$, short term (48 h) prophylactic hypothermia is not recommended to improve outcomes (Level IIB). 


\section{Glycemic Control}

After TBI there is a marked catecholamine surge, with cortisol release and glucose intolerance leading to significant hyperglycemia. Anaerobic metabolism of glucose and resulting acidosis in the brain may lead to neuronal dysfunction and cerebral edema. Impaired cerebrovascular regulation following TBI has also been implicated as a reason for poor outcome because of hyperglycemia [41]. Glucose-containing fluids should be avoided and blood sugar monitored to maintain levels between 4-8 $\mathrm{mmol} / \mathrm{L}$.

\section{Decompressive Craniectomy}

Decompressive craniectomy is a surgical procedure that involves removal of a large section of the skull. Craniectomy reduces ICP by giving extra space to the swollen brain, and it may quickly prevent brainstem herniation.

The DECRA (Decompressive Craniectomy) trial included patients who had refractory increased ICPs between 15 minutes and 1 hour of onset [42]. There were significantly fewer medical interventions to decrease ICP in patients treated with decompressive craniectomy. However, at 6-month follow-up, functional outcome was worse in the decompressive craniectomy group compared with the standard of care group.

The RESCUEicp (Randomized Evaluation of Surgery with Craniectomy for Uncontrollable Elevation of Intracranial Pressure) trial compared secondary decompressive craniectomy to optimal medical management [43]. At six months, decompressive craniectomy resulted in lower mortality than medical management, but higher rates of vegetative state and disability.

The results of these trials confirm that decompressive craniectomy may be a life-saving surgery, but that is comes at the expense of higher chances of severe disability among survivors. The latest BTF guidelines recommend a large frontotemporoparietal decompressive craniectomy, as opposed to a smaller one, to target reduced mortality and better neurological outcomes (Level IIA recommendation).

\section{Nutrition}

Early nutritional support is associated with better outcomes and enteral feeding has been found to be beneficial. The BTF recommends basal caloric replacement by at least the fifth day, and at most by the seventh day post-injury. Transgastric jejunal feeding may also reduce the risk of ventilator associated pneumonia. Patients with severe TBI have gastric feeding intolerance, which may be attributed to dysfunctional gastric emptying secondary to increased ICP and the use of opiates. Prokinetic agents, such as metoclopramide, may improve feeding tolerance.
The BTF recommends the replacement of $140 \%$ and $100 \%$ of the resting metabolic expenditure in non-paralyzed and paralyzed patients, respectively; however, lower caloric intake may also be beneficial.

\section{Anitibiotic Therapy}

Since TBI patients are more likely to receive invasive monitoring and therapeutic treatments, including mechanical ventilation, they are also more likely to be at increased risk for the development of infections. Sources of potential infections need to be identified and appropriate therapy should be instituted.

A common source of infection is invasive monitoring of ICP. The incidence of ICP device infection has been reported to range from $1 \%$ to $27 \%$ [44]. Most studies cited by the BTF guidelines that evaluated prophylactic antibiotic coverage in patients with TBI have shown little significant differences in infection rates. Another study that evaluated patients who received bacitracin flushes [45] showed a higher rate of infection among the intervention group. The current guidelines suggest the use of antibiotic-impregnated catheters [46] to reduce infection rates, although this is only a Level III recommendation.

There is limited available data to support the use of antibiotic prophylaxis in TBI, especially as data suggests that such therapy may predispose these patients to more severe infections. However, evidence for antibiotic therapy following penetrating TBI is robust, and therapy should be maintained for at least 7-14 days.

\section{Other Considerations}

Patients with TBI are at significant risk of experiencing thromboembolic events [47]. Options for prevention include mechanical (graduated compression stockings or intermittent pneumatic compression), pharmacological (low-dose or low-molecular-weight heparin) prophylaxis, or a combination of both. Pharmacological thromboprophylaxis is usually initiated 48-72 $\mathrm{h}$ after neurosurgical intervention and in the absence of other contraindications [48]. Additional care includes peptic ulcer prophylaxis, physiotherapy, and full hygienic care. The importance of both high quality perioperative intensive care and rehabilitation therapy in these patients cannot be overstated. Proper physiotherapy and postdischarge care in this patient population has been found to be an independent predictor of mortality and morbidity, as reported by Gupta and colleagues [49].

\section{Head Injury in Children}

Trauma resulting from falls or traffic accidents is even emerging as a leading cause of mortality in the pediatric age group. 
Table 3. Pediatric Glasgow Coma Scale

\begin{tabular}{|c|c|c|c|c|}
\hline & \multicolumn{2}{|c|}{$>1$ year } & $<1$ year & Score \\
\hline \multirow[t]{4}{*}{ Eye opening } & \multicolumn{2}{|c|}{ Spontaneously } & Spontaneously & 4 \\
\hline & \multicolumn{2}{|c|}{ To verbal command } & To shout & 3 \\
\hline & \multicolumn{2}{|c|}{ To pain } & To pain & 2 \\
\hline & \multicolumn{2}{|c|}{ No response } & No response & 1 \\
\hline \multirow[t]{7}{*}{ Motor response } & \multicolumn{2}{|c|}{ Obeys } & Spontaneous & 6 \\
\hline & \multicolumn{2}{|c|}{ Localizes pain } & Localizes pain & 5 \\
\hline & \multicolumn{2}{|c|}{ Flexion-withdrawal } & Flexion-withdrawal & 4 \\
\hline & \multicolumn{2}{|c|}{ Flexion-abnormal } & Flexion-abnormal & 3 \\
\hline & \multicolumn{2}{|c|}{ Extension } & Extension & 2 \\
\hline & \multicolumn{2}{|c|}{ No response } & No response & 1 \\
\hline & $>5$ years & $<2-5$ years & $0-23$ months & \\
\hline \multirow[t]{5}{*}{ Verbal response } & Oriented & Appropriate words & Smiles/coos appropriately & 5 \\
\hline & Disoriented/confused & Inappropriate words & Cries, consolable & 4 \\
\hline & Inappropriate words & Persistent cries/screams & Persistent inappropriate crying & 3 \\
\hline & Incomprehensible sounds & Grunts & Grunts, agitated, restless & 2 \\
\hline & No response & No response & No response & 1 \\
\hline
\end{tabular}

Children have an incompletely developed nervous system, and the prognostication of TBI is made more complex by the anatomical and physiological variations that distinguish children from adults.

In children, the head is larger in proportion to the body surface area, and stability is dependent on ligamentous rather than bony structures. Because of their smaller stature, children are at a significantly increased risk of direct brain injury, as their heads may be the site of initial impact and absorb a higher percentage of the forces applied. Although infants and young children may tolerate ICP increases better than adults because of open sutures, the presence of open fontanelles and sutures does not preclude the presence of elevated intracranial pressure, which may manifest as excessive irritability, crying, vomiting or, rarely, as electrocardiogram changes [50].

Assessments of neurological status are more complicated in the pediatric population because of difficulty in assessing responses to various stimuli. As such, the GCS is modified for use in pediatric patients (Table 3 ).

A recent study from Korea, published in 2017, found that age, surgical intervention, subdural hemorrhage, and diffuse axonal injury were predictors for poorer outcomes in the pediatric population [51].

\section{Conclusion}

Recent reviews of the literature have suggested that modern research may not have significantly improved outcomes in patients with severe TBI, although progress has been made in understanding the mechanism of injury and general hospital care. A lack of convincing evidence remains for many of the thera- peutic approaches used in various guidelines, even after several randomized controlled trials have evaluated specific components of these guidelines. The intent of establishing guidelines is to use evidence-based therapeutic approaches to reduce variations in patient management and improve functional outcomes. There is substantial evidence that the treatment of these patients in centers with protocol-driven management is associated with better outcomes [52]. The BTF guidelines, which are most widely used for the management of severe TBI patients, are a constantly evolving set of recommendations based on meta-analysis approaches and systematic reviews originating from clinical outcome studies.

A study of severe TBI patients in which the patients were treated according to the BTF guidelines has shown improvements in outcomes between 2001 and 2009. Better clinical outcomes are likely to be a consequence of a combination of improved prehospital and critical care [53]. Prompt interventions to limit secondary brain injury are essential to improve the longterm outcomes in this patient population.

\section{ORCID}

Hari Hara Dash, https://orcid.org/0000-0003-4020-5388

Siddharth Chavali, https://orcid.org/0000-0001-9361-0833 


\section{References}

1. Lee KS. Estimation of the incidence of head injury in Korea: an approximation based on national traffic accident statistics. J Korean Med Sci 2001; 16: 342-6.

2. Carney N, Totten AM, O’Reilly C, Ullman JS, Hawryluk GW, Bell MJ, et al. Guidelines for the management of severe traumatic brain injury, fourth edition. Neurosurgery 2017; 80: 6-15.

3. Bhagat H, Narang R, Sharma D, Dash HH, Chauhan H. ST elevation--an indication of reversible neurogenic myocardial dysfunction in patients with head injury. Ann Card Anaesth 2009; 12: 149-51.

4. Davison DL, Terek M, Chawla LS. Neurogenic pulmonary edema. Crit Care 2012; 16: 212.

5. Dash HH. Prehospital care of head injured patients. Neurol India 2008; 56: 415-9.

6. Chesnut RM, Marshall LF, Klauber MR, Blunt BA, Baldwin N, Eisenberg HM, et al. The role of secondary brain injury in determining outcome from severe head injury. J Trauma 1993; 34: 216-22.

7. Spaite DW, Bobrow BJ, Stolz U, Sherrill D, Chikani V, Barnhart B, et al. Evaluation of the impact of implementing the emergency medical services traumatic brain injury guidelines in Arizona: the excellence in prehospital injury care (EPIC) study methodology. Acad Emerg Med 2014; 21: 818-30.

8. Teasdale G, Jennett B. Assessment of coma and impaired consciousness. A practical scale. Lancet 1974; 2: 81-4.

9. Wijdicks EF, Bamlet WR, Maramattom BV, Manno EM, McClelland RL. Validation of a new coma scale: the FOUR score. Ann Neurol 2005; 58: 585-93.

10. Lin K, Dulebohn SC. Ranchos Los Amigos. Treasure Island (FL), StatPearls Publishing. 2017.

11. Davis DP. Prehospital intubation of brain-injured patients. Curr Opin Crit Care 2008; 14: 142-8.

12. Sobuwa S, Hartzenberg HB, Geduld H, Uys C. Outcomes following prehospital airway management in severe traumatic brain injury. S Afr Med J 2013; 103: 644-6.

13. Davis JW, Parks SN, Detlefs CL, Williams GG, Williams JL, Smith RW. Clearing the cervical spine in obtunded patients: the use of dynamic fluoroscopy. J Trauma 1995; 39: 435-8.

14. Bhalla T, Dewhirst E, Sawardekar A, Dairo O, Tobias JD. Perioperative management of the pediatric patient with traumatic brain injury. Paediatr Anaesth 2012; 22: 627-40.

15. Caricato A, Conti G, Della Corte F, Mancino A, Santilli F, Sandroni C, et al. Effects of PEEP on the intracranial system of patients with head injury and subarachnoid hemorrhage: the role of respiratory system compliance. J Trauma 2005; 58: 571-6.

16. Dunham CM, Cutrona AF, Gruber BS, Calderon JE, Ransom KJ, Flowers LL. Early tracheostomy in severe traumatic brain injury: evidence for decreased mechanical ventilation and increased hospital mortality. Int J Burns Trauma 2014; 4: 14-24.

17. Contant CF, Valadka AB, Gopinath SP, Hannay HJ, Robertson CS. Adult respiratory distress syndrome: a complication of induced hypertension after severe head injury. J Neurosurg 2001; 95: 560-8.

18. Fontanilla L. Dopamine raises ICP. Crit Care 2001; 5: 73300.

19. Sookplung P, Siriussawakul A, Malakouti A, Sharma D, Wang J, Souter MJ, et al. Vasopressor use and effect on blood pressure after severe adult traumatic brain injury. Neurocrit Care 2011; 15: 46-54.

20. SAFE Study Investigators, Australian and New Zealand Intensive Care Society Clinical Trials Group, Australian Red Cross Blood Service, George Institute for International Health, Myburgh J, Cooper DJ, et al. Saline or albumin for fluid resuscitation in patients with traumatic brain injury. N Engl J Med 2007; 357: 874-84.

21. Li N, Zhao WG, Zhang WF. Acute kidney injury in patients with severe traumatic brain injury: implementation of the acute kidney injury network stage system. Neurocrit Care 2011; 14: 377-81.

22. Perel P, Roberts I, Ker K. Colloids versus crystalloids for fluid resuscitation in critically ill patients. Cochrane Database Syst Rev 2013; (2): CD000567.

23. Chou A, Krukowski K, Jopson T, Zhu PJ, Costa-Mattioli M, Walter P, et al. Inhibition of the integrated stress response reverses cognitive deficits after traumatic brain injury. Proc Natl Acad Sci U S A 2017; 114: E6420-6.

24. Oddo M, Crippa IA, Mehta S, Menon D, Payen JF, Taccone FS, et al. Optimizing sedation in patients with acute brain injury. Crit Care 2016; 20: 128.

25. Sharshar T, Citerio G, Andrews PJ, Chieregato A, Latronico N, Menon DK, et al. Neurological examination of critically ill patients: a pragmatic approach. Report of an ESICM expert panel. Intensive Care Med 2014; 40: 484-95.

26. Balestreri M, Czosnyka M, Hutchinson P, Steiner LA, Hiler M, Smielewski P, et al. Impact of intracranial pressure and cerebral perfusion pressure on severe disability and mortality after head injury. Neurocrit Care 2006; 4: 8-13.

27. Nathens AB, Cryer HG, Fildes J. The American college of surgeons trauma quality improvement program. Surg Clin North Am 2012; 92: 441-54.

28. Cottenceau V, Masson F, Mahamid E, Petit L, Shik V, Sztark F, et al. Comparison of effects of equiosmolar doses of mannitol and hypertonic saline on cerebral blood flow and metabolism in traumatic brain injury. J Neurotrauma 2011; 28: 2003-12.

29. Tisdall MM, Smith M. Multimodal monitoring in traumatic brain injury: current status and future directions. Br J Anaesth 2007; $99: 61-7$.

30. Murr R, Schürer L. Correlation of jugular venous oxygen saturation to spontaneous fluctuations of cerebral perfusion pressure in patients 
with severe head injury. Neurol Res 1995; 17: 329-33.

31. Narotam PK, Morrison JF, Nathoo N. Brain tissue oxygen monitoring in traumatic brain injury and major trauma: outcome analysis of a brain tissue oxygen-directed therapy. J Neurosurg 2009; 111: 672-82.

32. de Lima Oliveira M, Kairalla AC, Fonoff ET, Martinez RC, Teixeira MJ, Bor-Seng-Shu E. Cerebral microdialysis in traumatic brain injury and subarachnoid hemorrhage: state of the art. Neurocrit Care 2014; 21: 152-62.

33. Clausen T, Alves OL, Reinert M, Doppenberg E, Zauner A, Bullock R. Association between elevated brain tissue glycerol levels and poor outcome following severe traumatic brain injury. J Neurosurg 2005; 103: 233-8.

34. Vespa PM, Nuwer MR, Nenov V, Ronne-Engstrom E, Hovda DA, Bergsneider M, et al. Increased incidence and impact of nonconvulsive and convulsive seizures after traumatic brain injury as detected by continuous electroencephalographic monitoring. J Neurosurg 1999; 91: 750-60.

35. Vespa PM, Miller C, McArthur D, Eliseo M, Etchepare M, Hirt D, et al. Nonconvulsive electrographic seizures after traumatic brain injury result in a delayed, prolonged increase in intracranial pressure and metabolic crisis. Crit Care Med 2007; 35: 2830-6.

36. Temkin NR, Dikmen SS, Wilensky AJ, Keihm J, Chabal S, Winn HR. A randomized, double-blind study of phenytoin for the prevention of post-traumatic seizures. A randomized, double-blind study of phenytoin for the prevention of post-traumatic seizures. N Engl J Med 1990; 323: 497-502.

37. Yang Y, Zheng F, Xu X, Wang X. Levetiracetam versus phenytoin for seizure prophylaxis following traumatic brain injury: a systematic review and meta-analysis. CNS Drugs 2016; 30: 677-88.

38. Sadaka F, Veremakis C. Therapeutic hypothermia for the management of intracranial hypertension in severe traumatic brain injury: a systematic review. Brain Inj 2012; 26: 899-908.

39. Andrews PJ, Sinclair HL, Rodriguez A, Harris BA, Battison CG, Rhodes JK, et al. Hypothermia for intracranial hypertension after traumatic brain injury. N Engl J Med 2015; 373: 2403-12.

40. O’Phelan KH, Merenda A, Denny KG, Zaila KE, Gonzalez C. Therapeutic temperature modulation is associated with pulmonary complications in patients with severe traumatic brain injury. World J Crit Care Med 2015; 4: 296-301.

41. Shi J, Dong B, Mao Y, Guan W, Cao J, Zhu R. Review: traumatic brain injury and hyperglycemia, a potentially modifiable risk factor. Oncotarget 2016; 7: 71052-61.

42. Cooper DJ, Rosenfeld JV, Murray L, Arabi YM, Davies AR, D’Urso P, et al. Decompressive craniectomy in diffuse traumatic brain injury. N Engl J Med 2011; 364: 1493-502.

43. Hutchinson PJ, Kolias AG, Timofeev IS, Corteen EA, Czosnyka M, Timothy J. Trial of decompressive craniectomy for traumatic intracranial hypertension. N Engl J Med 2016; 375: 1119-30.

44. Rebuck JA, Murry KR, Rhoney DH, Michael DB, Coplin WM. Infection related to intracranial pressure monitors in adults: analysis of risk factors and antibiotic prophylaxis. J Neurol Neurosurg Psychiatry 2000; 69: 381-4.

45. Aucoin PJ, Kotilainen HR, Gantz NM, Davidson R, Kellogg P, Stone B. Intracranial pressure monitors. Epidemiologic study of risk factors and infections. Am J Med 1986; 80: 369-76.

46. Wang X, Dong Y, Qi XQ, Li YM, Huang CG, Hou LJ. Clinical review: efficacy of antimicrobial-impregnated catheters in external ventricular drainage-a systematic review and meta-analysis. Crit Care 2013; 17: 234.

47. Denson K, Morgan D, Cunningham R, Nigliazzo A, Brackett D, Lane M, et al. Incidence of venous thromboembolism in patients with traumatic brain injury. Am J Surg 2007; 193: 380-3.

48. Scudday T, Brasel K, Webb T, Codner P, Somberg L, Weigelt J, et al. Safety and efficacy of prophylactic anticoagulation in patients with traumatic brain injury. J Am Coll Surg 2011; 213: 148-53.

49. Gupta D, Sharma D, Kannan N, Prapruettham S, Mock C, Wang J. Guideline adherence and outcomes in severe adult traumatic brain injury for the CHIRAG (collaborative head injury and guidelines) study. World Neurosurg 2016; 89: 169-79.

50. Dash M, Bithal PK, Prabhakar H, Chouhan RS, Mohanty B. ECG changes in pediatric patients with severe head injury. J Neurosurg Anesthesiol 2003; 15: 270-3.

51. Jeong HW, Choi SW, Youm JY, Lim JW, Kwon HJ, Song SH. Mortality and epidemiology in 256 cases of pediatric traumatic brain injury: Korean Neuro-trauma data bank system (KNTDBS) 2010-2014. J Korean Neurosurg Soc 2017; 60: 710-6.

52. Talving P, Karamanos E, Teixeira PG, Skiada D, Lam L, Belzberg H, et al. Intracranial pressure monitoring in severe head injury: compliance with brain trauma foundation guidelines and effect on outcomes: a prospective study. J Neurosurg 2013; 119: 1248-54.

53. Hesdorffer DC, Ghajar J. Marked improvement in adherence to traumatic brain injury guidelines in United States trauma centers. J Trauma 2007; 63: 841-7. 